\title{
MÉTODO GEOESTADÍSTICO DE RESTAURACIÓN DE IMÁGENES SATELITALES LANDSAT EN TERRITORIOS NUBLADOS
}

\author{
GEOESTATISTICAL METHOD OF SATELLITAL IMAGE \\ RESTORATION LANDSAT IN CLOUDY TERRITORIES
}

Victor Flores Benites ${ }^{1}$

\begin{tabular}{rr} 
Victor Flores Benites $^{1}$ \\
\hline $\begin{array}{l}\text { 1. Facultad de Ingeniería Eléctrica y Electrónica. Universidad Nacional de Ingeniería, Lima, Perú. } \\
\text { E-mail:suryavf@gmail.com }\end{array}$ \\
Recibido: 3 Diciembre \\
Aceptado: 15 Diciembre \\
ECorrespondencia del autor. Facultad de Ingeniería Eléctrica y Electrónica. Universidad Nacional de Ingeniería, Lima, Perú. \\
E-mail:suryavf@gmail.com
\end{tabular}

\section{RESUMEN}

Las imágenes satelitales son una fuente de importante de información en el estudio de la superficie terrestre. El resultado de su análisis es influenciado por las condiciones atmosféricas en las que fueron tomadas. La nubosidad es la interferencia más común y su presencia puede ser perjudicial al procesamiento. En el presente trabajo se busca eliminar nubosidad en las imágenes satelitales, para esto se realiza una estimación de la superficie debajo de las nubes. Se propone una solución basada en GNSPI, método geoestadístico ideado originalmente para remoción de huecos (regiones no escaneadas por el satélite) provocados por una falla en el sensor SLC del satélite Landsat-7. Se ha demostrado que existe una relación lineal entre imágenes tomadas en diferentes fechas. Por tanto, podemos realizar una estimación de la superficie debajo de las nubes a partir de una imagen sin nubes. La estimación calculada (predicción temporal) no es completa ya que no considera las variaciones locales del suelo, por lo que es necesario una segunda estimación. La predicción de las variaciones locales es realizada mediante un método geoestadístico: Kriging. La principal ventaja de este método geoestadístico frente a los deterministas es que tiene buenos resultados en superficies heterogéneas. Partimos del supuesto que pixeles similares muestran patrones similares en la variación espectral entre diferentes fechas. Por lo tanto, se puede obtener una buena estimación si se utiliza exclusivamente los pixeles similares para la predicción. La estimación final es la suma de la estimación temporal más la estimación local..

Palabras claves: remoción de nubes; GNSPI; geoestadística; kriging; pixeles similares. 


\begin{abstract}
Satellite images are an important source of information in the study of the earth's surface. The result of their analysis can be influenced by atmospheric conditions in which they were taken. Cloudiness is the most common interference and its presence can be detrimental to processing. The objective of this study is to eliminate clouds on satellite imagery. Consequently, a surface estimate beneath the clouds is carried out. A method based on GNSPI is proposed. This is a geostatistical method originally devised for removal of voids (regions scanned by the sate1lite) caused by a fault in the SLC sensor Landsat-7 solution. It has been shown that there is a linear relationship between images taken at different dates. Therefore, we can make an estimate of the surface beneath the clouds from an image without clouds. The calculated estimate (temporal prediction) is not complete and does not consider local variations soil, so a second estimate is needed. The prediction of local variations is performed using a geostatistical method: Kriging. The main advantage of this geostatistical method over the deterministic is that it has good results on heterogeneous surfaces. We assume that similar pixels show similar patterns in the spectral variation between different dates. Therefore, one can get a good estimate if used exclusively similar pixels for prediction. The final estimate is the sum on the temporal estimate and the local estimate.
\end{abstract}

Keywords: clearing clouds; GNSPI; geostatistics; kriging; Similar pixels.

\section{INTRODUCCIÓN}

La presencia de nubes en las imágenes capturadas por satélites es un problema común: el 98\% de las imágenes las contiene. Por lo general se suele elegir un umbral de nubosidad, las imágenes que poseen un porcentaje de nubosidad menor al umbral se procesan sin tomar en cuenta que existen nubes en la escena, trayendo consigo imperfecciones en los resultados. Una mejor solución es la segmentación de las nubes para luego no contarlas en el procesamiento, obteniendo resultados más fiables y dando la posibilidad de que el umbral de nubosidad sea mayor, pero en muchas ocasiones la pérdida de información por la segmentación es crítica. La estimación de la superficie debajo de las nubes puede ser una mejor solución. Se han realizado diversos estudios de cómo realizarla, en general las diversas soluciones se pueden clasificar en dos: métodos determinísticos y métodos geoestadísticos

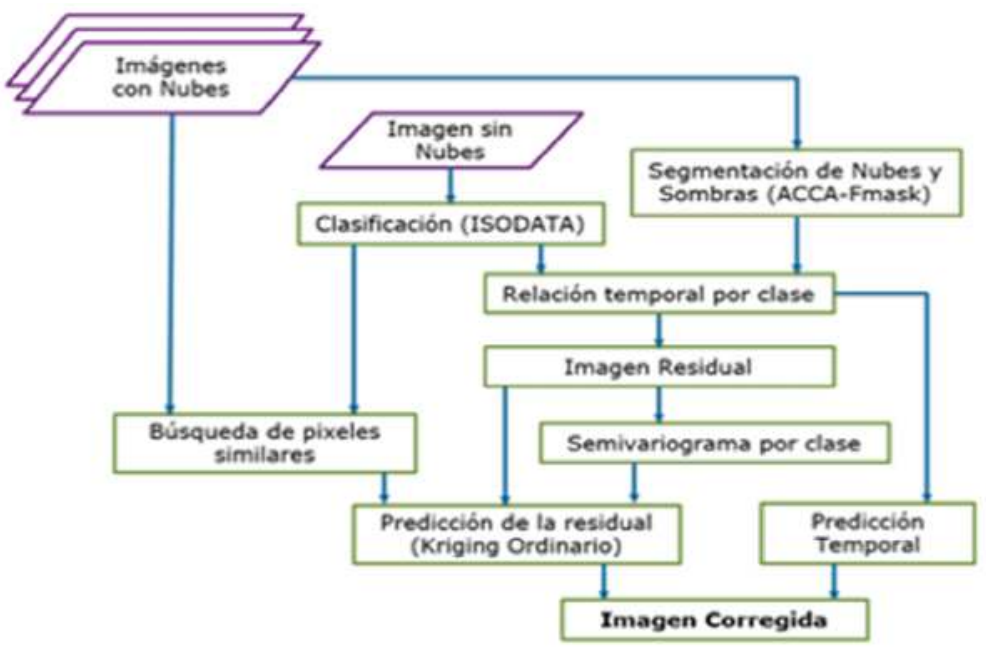

Figura. 1. Esquema general del Algoritmo. Se toma como base GNSPI (2) 
La ventaja de utilizar los métodos determinísticos es que en el procesamiento solo se utiliza la imagen a reparar, dando una solución sencilla y con baja carga computacional. En este caso, la familia de soluciones más comunes y simples son las interpolaciones por aproximación a funciones, aquí se estima los píxeles faltantes (correspondientes a nubes) usando los píxeles vecinos; esta solución dan malos resultados a media resolución y peores resultados en alta resolución. Una mejor solución es NSPI (1) (una solución popular de rellenado de huecos de imágenes Landsat-7), este enfoque fue exitoso al predecir características pequeñas del paisaje, pero al ser un método determinista no pudo introducir incertidumbre a la estimación.

La otra familia de soluciones son los geoestadísticos, su principal ventaja es tomar en cuenta la distribución espacial de la varianza (semivariograma). Los resultados obtenidos por estos algoritmos suelen ser mejores para imágenes de alta concentración de detalle, pero resulta ser poco eficiente en entornos suaves (como líneas de carretera). La razón de esta debilidad de los métodos geoestadísticos puede ser la suposición de estacionalidad intrínseca que no siempre es válido especialmente para los paisajes homogéneos (2).

Teniendo en cuenta la ventaja que poseen los algoritmos deterministas y geoestadísticos, en el presente trabajo se propone una solución que fusiona ambos enfoques. Para esto se toma de modelo la solución presentada por Xiaolin Zhu, Desheng Liu y Jin Chen en "A new geostatistical approach for filling gaps in Landsat ETM+ SLC-off images" (2): GNSPI. Este método, al igual que el NSPI, fue presentado para solucionar un problema de pixeles no escaneados del satélite Landsat-7.

\section{DESCRIPCIÓN DEL ALGORITMO}

Para la remoción y llenado de las nubes, el algoritmo utiliza varias imágenes auxiliares que fueron tomadas en la misma área. Una de estas imágenes será libre de nubes, a la que denominaremos TM0. Las siguientes imágenes contendrán nubes y serán reparadas, su denominación será TM1, TM2.

Se ha demostrado que existe una relación lineal entre pixeles correspondiente a una misma clase de superficie de una misma banda tomadas en diferentes fechas (3). De esta relación podemos obtener una predicción de la escena para cualquier fecha cercana a la captura de TM0 (2). Pero esta relación solo toma en cuenta variaciones globales de la superficie y no las variaciones locales que son propias de la naturaleza del suelo. De este análisis podemos concluir que nos encontramos ante dos problemas: el cálculo de la variación global y variación local. La variación global es calculada con una regresión lineal aprovechando la relación entra las imágenes de diferentes fechas (3), al resultado de este cálculo lo denominaremos predicción temporal. El cálculo de la variación local es realizado por el método geoestadístico Kriging y su resultado la denominaremos predicción residual. En la Figura 1 se muestra un esquema general del algoritmo, en las siguientes secciones cada paso será explicado a detalle.

\section{A. Segmentación de Nubes y Sombras}

Los algoritmos de reconocimientos de nubes son muy variados, siendo uno de lo más difundidos ACCA (4) (5). El algoritmo clasifica la escena en posibles regiones con nubes (ambiguas), regiones con nubes calientes y frías utilizando el índice NDSI (índice de nieve), temperatura y relación de la reflectancia entre bandas. Las regiones marcadas como ambiguas entran en un segundo procesamiento en donde los umbrales dependen de la temperatura de la escena, de la presencia de nieve y regiones desérticas.

ACCA no localiza las sombras de las nubes, por lo cual fue necesario el uso de otro algoritmo para su segmentación. Se utilizó Fmask para este fin (6). Este algoritmo utiliza el modelo de elevación digital de la Banda 4, en el que realiza una transformación morfológica de rellenado. Si la diferencia entre la transformada y la imagen original es mayor a un umbral, 0.02 (6), se clasificará el pixel como sombra.

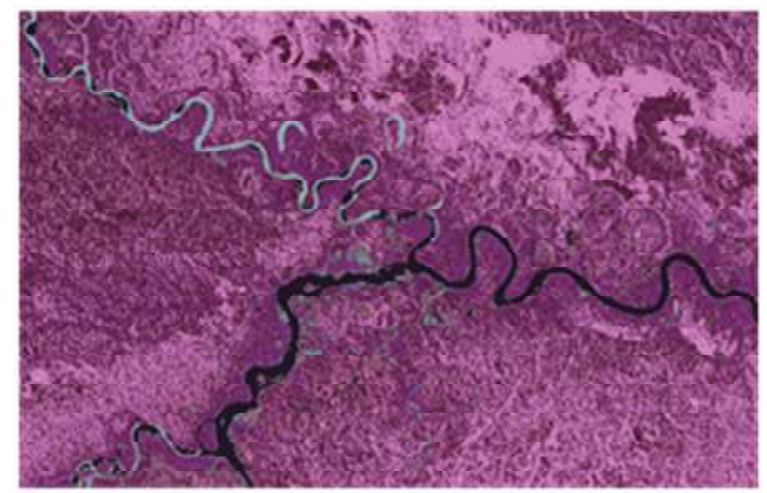

Figura. 2. Resultado de la clasificación por ISODATA

\section{B. Clasificación de la escena (Clustering)}

Para que el algoritmo se ejecute correctamente es necesario una buena clasificación. Se opta por el uso de una técnica no supervisada de clasificación, ISO- 
DATA (7), para que el programa no requiera de una intervención adicional en el procesamiento. Este algoritmo divide, fusiona y elimina grupos según parámetros previamente asignados. La división de un grupo es efectuada cuando este posee una desviación estándar (dispersión) superior a un umbral asignado. La fusión de grupos es efectuada cuando la distancia euclidiana de los centros de dos clusters es menor a un valor asignado. La eliminación de grupos se da cuando este posee un número reducido de miembros.

La clasificación de escena es solo realizada en la imagen TM0 debido a que en la imagen TM1 no es posible conocer la configuración de la superficie debajo de las nubes. La clasificación calculada es usada en los siguientes pasos para la imagen TM1.

\section{Calculo de la Relación Temporal}

La relación lineal entre las imágenes TM0 y TM1 es planteada en la Ecuación 1 (1), en donde R es la reflectancia en la posición $\mathrm{s}$ y banda $\mathrm{b}$ en el instante t1 o t2. Los coeficientes $a_{k}$ y $b_{k}$ son calculados con regresión lineal.

$$
\hbar\left(s, t_{2}, b\right)=\boldsymbol{d}_{k} \hbar\left(s, t_{1}, b\right)+t_{k}
$$

Esta relación no toma en cuenta las variaciones locales en el tiempo, por lo que tendremos un error de predicción. El error de predicción es calculado con la Ecuación 2 en donde se resta la reflectancia real de la superficie menos la reflectancia estimada en la Ecuación 1. Al resultado la denominaremos imagen residual.

$$
\mathrm{f}(s, b)=R\left(s, t_{2}, b\right)-\hbar\left(s, t_{2}, b\right)
$$

El error de predicción no puede ser calculado en áreas con presencia de nubes ya que no tenemos datos de la superficie debajo de ellas. El objetivo de los siguientes pasos será la estimación del valor residual de la superficie debajo de las nubes.

\section{Semivariograma}

El semivariograma es la función que describe la variabilidad espacial de un fenómeno de interés. Matemáticamente, es la representación gráfica de las varianzas en función de la distancia que separa a las muestras, es calculada mediante la Ecuación 3 en donde $\mathrm{Z}$ es una característica medida en el punto si.

$y(h)=\frac{1}{2 N(h)}{ }_{i=1}^{N(h)}\left[Z\left(s_{i}\right)-Z\left(s_{i}+h\right)\right]^{2}$
El semivariograma es más útil si es relacionado a una función matemática, en el presente trabajo se aproxima al modelo exponencial (Ecuación 4).

$$
y(h)=(s-n)\left(1-e^{\frac{\mathrm{d} n}{r}}\right)+n
$$

\section{E. Ajuste de Curva por Algoritmo Genético}

El ajuste del modelo del semivariograma es realizado por un Algoritmo Genético Adaptativo planteado por Jiao Lei-yin, Lei Hong-zhuan (8). Esta variación de AG tiene como particularidad que la función de adaptación, la probabilidad de cruce y de mutación depende de la generación en la que nos encontremos. Estas obedecen a las Ecuaciones 5, 6 y 7 respectivamente.

$$
\begin{gathered}
f^{r}=e^{\frac{l s}{k} ;} T_{\mathrm{k}}=\frac{T_{0}}{k} \\
F_{C}=\left\{\begin{array}{cc}
\mathrm{P}_{1}+\frac{k_{1}\left(f_{\max }-f\right)}{f_{\max }-f_{\text {avg }}} & f \$ f_{\text {avg }} \\
k_{2} & f 1 f_{\text {avg }}
\end{array}\right. \\
P m=\left\{\begin{array}{cc}
\mathrm{p}_{2}+\frac{k_{3}\left(f_{\max }-f^{\prime}\right)}{f_{\max }-f_{\text {avg }}} & f^{\prime} \$ f_{\text {avg }} \\
k_{4} & f^{\prime} 1 f_{\text {avg }}
\end{array}\right.
\end{gathered}
$$

Donde, $f_{\max }$ es la máxima adaptación de la población, $f_{\text {avg }}$ es la adaptación promedio, $f$ es la adaptación máxima entre los dos individuos a cruzarse, $f^{\prime}$ es la adaptación de un individuo, $k$ es la generación actual, 1s es el error cuadrático medio del ajuste de curvas actual. $\mathrm{T}_{0}, \mathrm{k}_{1}, \mathrm{k}_{2}, \mathrm{k}_{3}, \mathrm{k}_{4}, \mathrm{P}_{1}, \mathrm{P}_{2}$ son constantes.

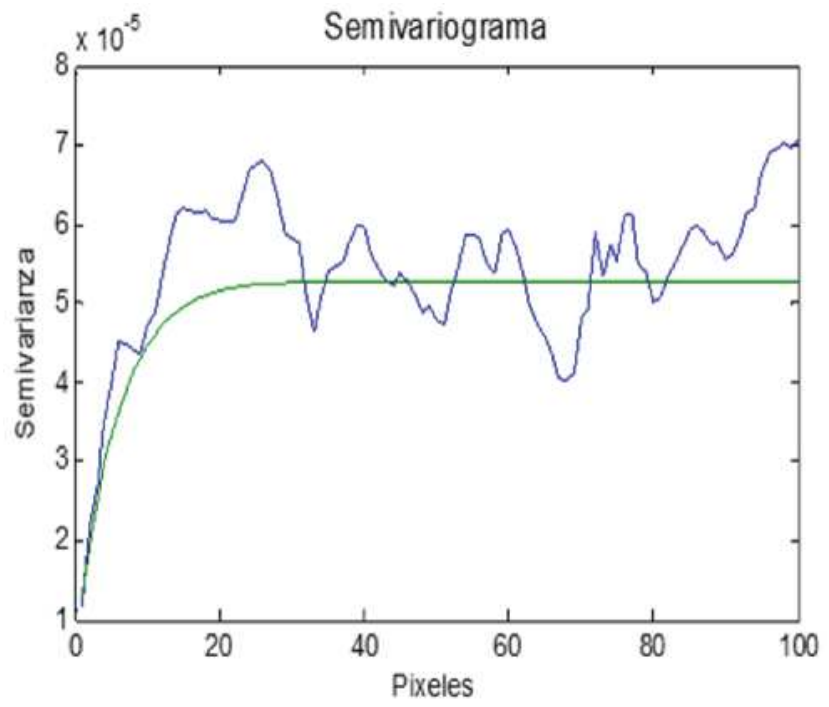

Figura. 3. Semivariograma. La curva experimental es mostrada en color azul y el resultado del ajuste de curvas en verde. 


\section{F. Pixeles similares}

Pixel similar es un concepto introducido en "A simple and effective method for filling gaps in Landsat ETM plus SLC-off images" (1). La similitud espectral es definida con la Ecuación 8. Si dos pixeles cumplen con la Ecuación 9 se dicen que son similares. En las ecuaciones: RMSD (root mean square difference) es la raíz cuadrada de la media del cuadrado de diferencias entre valores de reflectancia de dos pixeles por banda; $\mathrm{n}$ es el número de bandas; $\mathrm{m}$ es el número de grupos (clusters); $\sigma(b)$ es la varianza por banda.

$$
\begin{gathered}
R M S D_{i}=\sqrt{\frac{/_{b=1}^{n}\left(R\left(s_{i}, b\right)-R\left(s_{t}, b\right)\right)^{2}}{n}} \\
R M S D_{i} \#\left[{ }_{b=1}^{n} \mathbf{v}(b) X^{2} / M\right] / M
\end{gathered}
$$

Partimos del supuesto que los pixeles especialmente adyacentes con alta similitud espectral tienen patrones cambiantes similares. Entonces, para predecir el

valor residual de un pixel correspondiente a la superficie debajo de la nube, se buscarán los pixeles que le son similares. Esta búsqueda se realiza en las imágenes en donde esos pixeles no se encuentren cubiertos por nubes.

\section{G. Kriging}

El Kriging es un método geoestadístico de estimación de puntos que utiliza un modelo de variograma para la obtención de datos. Sea el campo aleatorio $\mathrm{Z}(\mathrm{s})$ : sd $\mathrm{D} f \mathrm{R}^{\mathrm{d}}$ donde se ha observado el atributo $\mathrm{Z}$ en las ubicaciones $s_{1}, s_{2}, \ldots, s_{n}$ y se desea predecir dicho atributo en una ubicación no observada, $\mathrm{s}_{0}$, basándose en los valores obtenidos en las muestras hechas.

$$
z\left(x_{0}\right)=\prod_{i=1}^{n} \mathbf{m}_{i} Z\left(s_{i}\right)
$$

Un método de cálculo de los factores de ponderación $(\lambda i)$ es el Kriging Ordinario, en donde se asume que la variable es estacionaria con varianza conocida y media desconocida. De acuerdo a estas premisas se puede demostrar que $\lambda \mathrm{i}$ cumple la condición 15.

a)

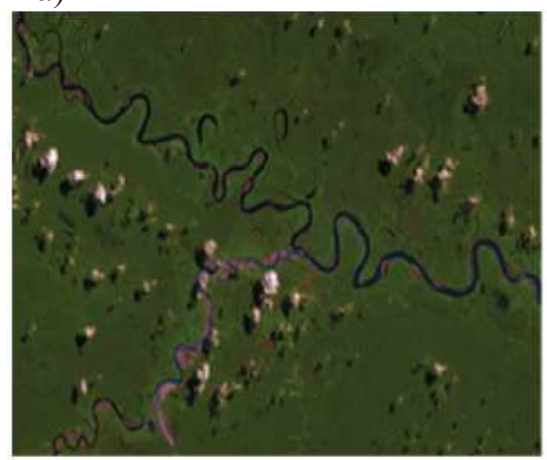

d)

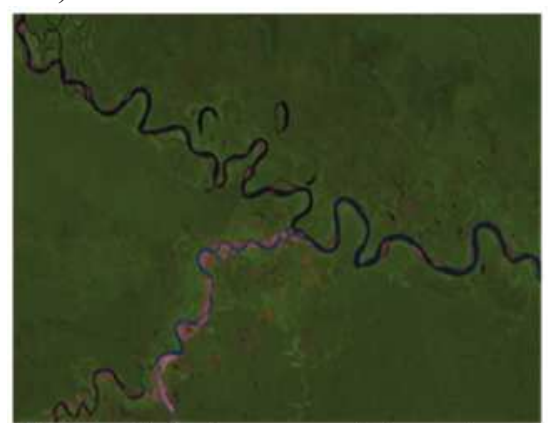

b)

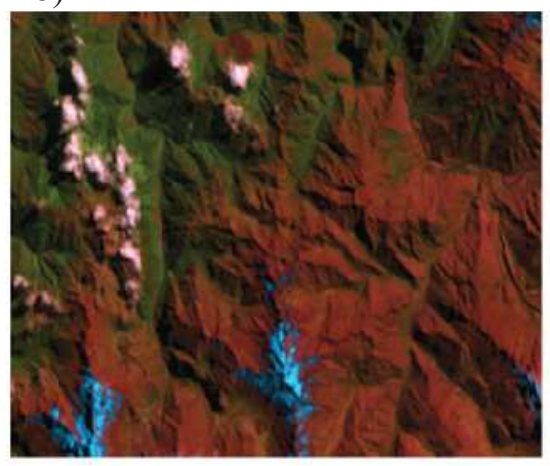

e)

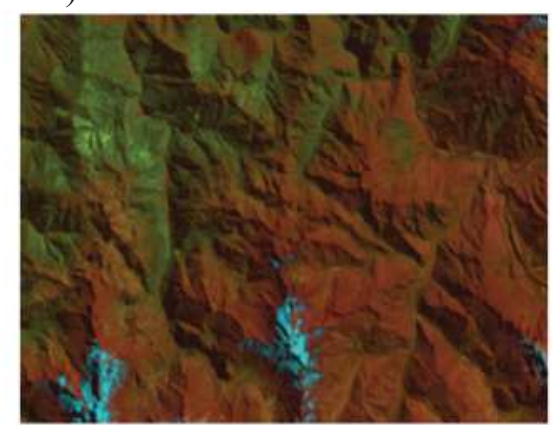

c)

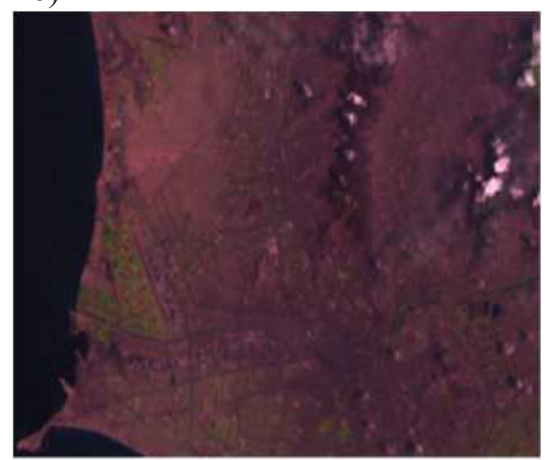

f)

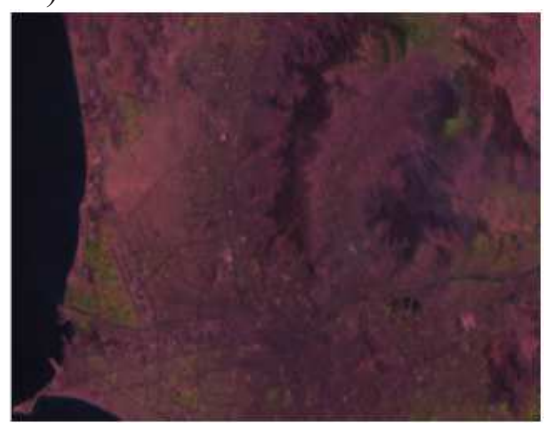

Figura. 4 Imágenes en falso color, modelo 543. En las imágenes a), b) y c) se muestran las imágenes originales. En las imágenes d), e) y f) se muestra el resultado del algoritmo. La imagen a) fue capturada en Loreto, la imagen b) en Cusco, y la imagen c) en Lima.

$$
\prod_{i=1}^{n} \mathbf{m}_{i} \operatorname{Cov}\left(s_{i}-s_{j}\right)-\mathbf{n}=\operatorname{Cov}\left(s_{i}-s_{j}\right)
$$


Para predecir el valor residual del pixel pi de la imagen TM1, se buscarán que pixeles son similares a él en la imagen TM0 (en donde tenemos información de su naturaleza espectral) y se usarán estos para el cálculo de los parámetros $\lambda i$. Un mejor resultado se obtiene si adicional a la búsqueda en la imagen TM0 se realiza otra búsqueda en una imagen TM2 en donde no existan nubes en la ubicación del pixel pi. Encontrados los pixeles similares en TM0 y TM2, se utilizarán los pixeles similares en ambas imágenes para el cálculo de los parámetros $\lambda \mathrm{i}$.

\section{DISCUSIÓN DE RESULTADOS}

El algoritmo fue probado en imágenes capturadas por el satélite Landsat-5, los ensayos fueron realizados en las primeras 5 bandas. Los resultados son mostrados en falso color con el modelo 543 en la Figura 4.

Durante las pruebas se ha encontrado que una omisión de pixeles en la clasificación de nubes y sombras altera seriamente la respuesta, ante este problema se prefirió utilizar umbrales que puedan exagerar la detección. Además se utilizó el operador morfológico de dilatación sobre la salida de ACCA y Fmask para garantizar que las nubes y sombras sean segmentadas en su totalidad.

La calidad del resultado del algoritmo depende principalmente del clustering, del ajuste de curvas del semivariograma y de la selección de pixeles a utiliza en Kriging. Una pobre clasificación de la superficie conlleva a la falta de detalles en el resultado pero una clasificación minuciosa eleva el tiempo de ejecución. De acuerdo a los ensayos, recomendamos un número inicial de 10 clousters.

Se encontraron casos en que el semivariograma experimental tenía un rango menor a 10 pixeles $(300$ metros), generando problemas en el ajuste de curvas. Para hacer frente a este problema, el ajuste fue realizado con muestras de hasta 50 pixeles de distancia. Se obtiene un buen desempeño del AG con la normalización del semivariograma y la acotación de la búsque- da del Sill, Nugget y Rango. En la curva normalizada, el rango de búsqueda del Sill fue de 0.7 a 1 , del Nugget de 0 a 0.2 y del Rango de 5 a 30 . El resultado del ajuste es mostrado en la Figura 3.

La predicción residual es realizada con Kriging mediante la suma ponderada mostrada en la ecuación 14 , en donde los parámetros $\lambda i$ son calculados con la ecuación 15 , esta ecuación utiliza los pixeles similares que son definidos mediante la ecuación 9. La búsqueda de los pixeles similares es realizada por cada pixel de la imagen, en consecuencia, la carga computacional añadida es muy alta. El tiempo de ejecución de Kriging también es grande: aumenta exponencialmente con el tamaño de la imagen y la cantidad de nubes presentes. La carga computacional añadida es tan grande puede representar el $90 \%$ del total. Para resolver estos problemas se ideó una estrategia en la búsqueda de los pixeles similares. Se definió un número máximo de pixeles similares y su búsqueda fue radial al pixel analizado con tal de asegurarnos de seleccionar a los vecinos cercanos, además se definió un rango máximo de búsqueda. El número máximo de pixeles similares disminuyó en gran medida el tiempo necesario para la ejecución de Kriging y el rango máximo de búsqueda disminuyó el tiempo necesario para definir los pixeles similares.

\section{CONCLUSIONES}

Con el algoritmo propuesto se ha conseguido realizar una correcta remoción de nubes, Kriging generó una buena predicción en regiones homogéneas y heterogéneas. El algoritmo tiene buenos resultados pero es débil ante los fallos de la segmentación de nubes y sombras, por lo que una exageración en su reconocimiento es recomendable. Además, el costo de un buen resultado es el incremento del tiempo de ejecución. Se recomienda realizar un análisis del tiempo de ejecución versus resultado para la aplicación específica en el que se desea utilizar el algoritmo. Se recomienda realizar un estudio de paralelización del algoritmo para tener un mejor resultado en tiempo de ejecución. 


\section{BIBLIOGRAFÍA}

1. Chen, J., Zhu, X., Vogelmann, J. E., Gao, F., \& Jin, S. M. (2011). "A simple and effective method for filling gaps in Landsat ETM plus SLC-off images".

2. Xiaolin Zhu, Desheng Liu, Jin Chen. (2012). "A new geostatistical approach for filling gaps in Landsat ETM+ SLC-off images".

3. Zhu, X., Chen, J., Gao, F., \& Masek, J. G. (2010). "An enhanced spatial and temporal adaptive reflectance fusion model for complex heterogeneous regions".

4. Richard R. Irish. "Landsat 7 Automatic Cloud Cover Assessment". Science Systems and Applications, Inc. NASA's Goddard Space Flight Center, Greenbelt, Maryland.

5. Richard R. Irish, John L. Barker, Samuel N. Goward, and Terry Arvidson. "Characterization of the Landsat-7 ETM+ Automated Cloud-Cover Assessment (ACCA) Algorithm". Photogrammetric Engineering \& Remote Sensing.

6. Zhe Zhu, Curtis E. Woodcock. "Object-based cloud and cloud shadow detection in Landsat imagery". Center for Remote Sensing, Department of Geography and Environment, Boston University.

7. Memarsadeghi N., Mount D. M., Netanyahu N. S., \& Le Moigne J. (2006). “A Fast Implementation of the ISODATA Clustering Algorithm".

8. Jiao Lei-yin, Lei Hong-zhuan. "The Application of Improved Genetic Algorithm in Fitting the Spatial Variogram". 2011 International Conference on Computer Science and Network Technology.

9. Lazaros Oreopoulos, Michael J. Wilson, Tamás Várnai. (2011). "Implementation on Landsat Data of a Simple Cloud-Mask Algorithm Developed for MODIS Land Bands".

10. Qi Chen, Peng Gong. (2004). "Automatic Variogram Parameter Extraction for Textural Classification of the Panchromatic IKONOS Imagery" 